\title{
Regular exercise and the trajectory of health-related quality of life among Taiwanese adults: a cohort study analysis 2006-2014
}

Huan-Cheng Chang ${ }^{1,2}$, Jersey Liang ${ }^{3}$, Hui-Chuan Hsu ${ }^{4,5^{*}}$ (D), Sung-Kai Lin ${ }^{6}$, Ting-Huan Chang ${ }^{7}$ and Shu-Hui Liu ${ }^{8}$

\begin{abstract}
Background: Physical activity is related to health-related quality of life, but little evidence from multiple waves of panel data in Asian countries area available. This study aims to explore the impacts of different degree of regular exercise on the trajectories of physical and mental dimensions of health-related quality of life (HRQOL) for communitydwelling Taiwanese adults during 2006-2014.

Methods: Data were derived from the "Landseed Integrated Outreaching Neighborhood Screening (LIONS)" study, with 6182 adults enrolled at the baseline and subsequently followed up to three times till 2014. Linear mixed-effects modeling approach was employed to evaluate the growth curve models of HRQOL (with 16,281 observations) by linear \& quadratic time effects, regular exercise (5-level moderate-intensity physical activity), and major influential factors of HRQOL.

Results: Regular exercise showed significantly positive dose-response effects on physical HRQOL ( $\beta=1.27 \sim 2.54)$, and regular exercise of $150 \mathrm{~min}$ or more showed positive effects on mental HRQOL ( $\beta=1.55 \sim 2.03$ ). Besides, irregular exercise could also improve both physical and mental HRQOL $(\beta=1.27 \& \beta=0.87)$. However, such effects were not significant over time (at time slope) on HRQOL. In addition, physical and mental HRQOL improved across time ( $\beta=1.01$ and 1.49 , respectively), but the time quadratic effect would significantly offset a little bit on physical dimension $(\beta=-0.22)$. Moreover, being female, increasing age, living alone, or poorer health status were related to lower physical HRQOL; and being younger, living alone, or poorer health status were associated with lower mental HRQOL.

Conclusions: The positive dose-response relationship between regular exercise and HRQOL or its domains was demonstrated for community-dwelling Taiwanese adults. Thus, a regular exercise habit (better $\geqq 150$ min per week) is advised for community-based healthcare professionals and the government to incorporate into health promotion strategies and plans.
\end{abstract}

Keywords: Health-related quality of life, Exercise, Physical activity, Cohort study

\footnotetext{
* Correspondence: gingerhsu@seed.net.tw

${ }^{4}$ School of Public Health, Taipei Medical University, No.250, Wuxing Street,

Taipei 11031, Taiwan, Republic of China

${ }^{5}$ Research Center of Health Equity, College of Public Health, Taipei Medical

University, No.250, Wuxing Street, Taipei 11031, Taiwan, Republic of China

Full list of author information is available at the end of the article
}

(c) The Author(s). 2019 Open Access This article is distributed under the terms of the Creative Commons Attribution 4.0 International License (http://creativecommons.org/licenses/by/4.0/), which permits unrestricted use, distribution, and reproduction in any medium, provided you give appropriate credit to the original author(s) and the source, provide a link to the Creative Commons license, and indicate if changes were made. The Creative Commons Public Domain Dedication waiver (http://creativecommons.org/publicdomain/zero/1.0/) applies to the data made available in this article, unless otherwise stated. 


\section{Introduction}

Numerous studies contend that regular exercise or health promoting or leisure time physical activity (PA) has a variety of health benefits. Some researchers assert that regular exercise can effectively lower the prevalence of many diseases [1-3], and postpone the onset of disabilities [4]. In addition, health promoting PA can also improve physical health [2], and mental health [5-11].

Health-related quality of life (HRQOL) has emerged as an important multidimensional concept in clinical and public health research in past decades [9, 12, 13], and been recognized as a desired health outcome for evaluating preventive or therapeutic health plans $[14,15]$. The relationship between PA (including exercise) and HRQOL has been widely explored, and consistently demonstrated to be a positive association across age groups and countries [8, 16, 17]. Higher levels of PA were related to better HRQOL [18]. Higher dose of exercise exerted larger improvements in physical and mental quality-of-life [10].

Risk and protective factors have been found to be related to HRQOL. Older age, female gender, living alone, lower education, and poorer physical health (i.e., more diseases or medications) would be associated with worse HRQOL [19-21]. Moreover, unhealthy behaviors (i.e., smoking, alcohol drinking, betel-nut chewing) and mental/psychiatric disorders were related to poorer HRQOL [22-26].

Although the impacts of PA on HRQOL have received great attentions, there are research gaps in the literature. First, the vast majority of studies on the relations of PA to HRQOL have been conducted in Western countries, whereas fewer studies were carried out in Asian nations. Because of the differences in life style, healthcare, and social determinants between Western nations and nonWestern nations, further replication of prior observations are warranted. Second, although past studies explored the effects of different intensity or time of PA on physical or mental health, most of the findings of positive association of PA with HRQOL were usually found in cross-sectional studies or in the only two-wave longitudinal studies $[8,11,27-31]$. The time effect of physical activities on HRQOL was little explored, and the timevarying covariates were not considered.

This study aims to explore the impacts of different degree of regular exercise on the trajectories of physical and mental dimensions of HRQOL for adults who participated in a multiple-wave community-based health screening program in Taiwan during 2006-2014.

\section{Methods}

\section{Study design and sample population}

Data were derived from a cohort study "Landseed Integrated Outreaching Neighborhood Screening (LIONS)" which has been conducted by the Landseed International Hospital in Taiwan to surveil chronic diseases (e.g., type 2 Diabetes mellitus, hypertension, cancers, cardiovascular diseases, osteoporosis, gout, psychiatric disorder) and relevant risk factors routinely for community-dwelling Taiwanese adults aged 30 or over. A sample was drawn by the probability-proportional-to-size sampling from the registered households of the Pingzhen district of Taoyuan City, Taiwan. The districts was chosen because it is the primary area of the patients cared by Landseed International Hospital from the LION project. They were invited to receive on-site health screening examinations to investigate four regulated cancers and other prevailing diseases, and a face-to-face survey interview. The design and implementation of LIONS was delineated in depth in a previous study [32].

LIONS was initiated in 2006 and enrolled 6182 individuals in nine admission cohorts during 2006-2014 (Table 1). All enrollees, with the free will to partake in any follow-up health screening, would be contacted 2 years after the baseline. Follow-ups were made for those admission cohorts at varying intervals, ranging from 1 to 3 years. Consequently, there were up to 4 waves for each admission cohort, and the observations by waves were 6182, 4268, 3424 and 2407 participants, respectively. In total, 16,281 observations were included in the analyses. By examining the goodness of fit, the sample was significantly different from the population: the sample was consisted of more females, older, and lower educated persons.

\section{Measures}

\section{The outcome variable}

Health-related quality of life was measured by Taiwan version of Medical Outcomes Study short-form 36-item (SF-36) questionnaire [33], which was validated from the original SF-36 questionnaire [34, 35]. The SF-36 questionnaire evaluates eight constructs of generic health and well-being, including physical functioning, role limitations due to physical problems, bodily pain, general health, vitality, social functioning, role limitation due to emotional problems, and mental health [36]. Based on the scores of eight constructs, two overall scores of physical component summary (PCS) and mental component summary (MCS) were computed, ranging from 0 to 100 [37]. Higher PCS and MCS scores indicate better perceived physical and mental health, respectively. The Cronbach's alpha of the 8 domains in Taiwanese population ranged from 0.76 to 0.92 , except the internal consistency of social function was only 0.65 [33].

\section{Health behaviors}

Health behaviors involved several time-varying variables. The primary indicator, regular exercise habit, was measured 
Table 1 Enrollment in health screening and subsequent participation in follow-ups

\begin{tabular}{|c|c|c|c|c|c|c|c|c|c|}
\hline \multirow{2}{*}{$\begin{array}{l}\text { Admission } \\
\text { cohorts }\end{array}$} & \multicolumn{9}{|l|}{ Year } \\
\hline & 2006 & 2007 & 2008 & 2009 & 2010 & 2011 & 2012 & 2013 & 2014 \\
\hline 2006 & $1605^{a}$ & & $1137^{b}$ & & & $874^{c}$ & & & $649^{d}$ \\
\hline 2007 & & $2068^{a}$ & & $1483^{b}$ & & $1155^{c}$ & & & $782^{d}$ \\
\hline 2008 & & & $1424^{a}$ & & $1066^{b}$ & $949^{c}$ & & & $654^{d}$ \\
\hline 2009 & & & & $676^{a}$ & & $425^{b}$ & $321^{c}$ & & $269^{d}$ \\
\hline 2010 & & & & & $128^{\mathrm{a}}$ & & $63^{b}$ & $59^{c}$ & $53^{d}$ \\
\hline 2011 & & & & & & $156^{\mathrm{a}}$ & & $71^{b}$ & $66^{c}$ \\
\hline 2012 & & & & & & & $56^{\mathrm{a}}$ & & $23^{b}$ \\
\hline 2013 & & & & & & & & $39^{a}$ & \\
\hline 2014 & & & & & & & & & $30^{\mathrm{a}}$ \\
\hline
\end{tabular}

${ }^{a}$ Enrollment at baseline ( $N=6182$, across admission cohorts)

${ }^{b} 1^{\text {st }}$ follow-up participation ( $n=4268$, across admission cohorts)

c $2^{\text {nd }}$ follow-up participation ( $n=3424$, across admission cohorts)

$\mathrm{d}_{3}{ }^{\text {rd }}$ follow-up participation ( $n=2407$, across admission cohorts)

according to 2008 Physical Activity Guidelines for Americans (PAGA) [38]. PAGA suggests physical activity level can be measured as four levels: inactive, low activity $(<150$ min), medium activity (150-299 min), and high activity ( $\geqq 300 \mathrm{~min}$ ). The participants were asked about the frequency of doing exercise and the time in minutes spent on exercise per week. The questions were "On average, how many times do you have the regular exercise per week, and how many minutes do you spend on doing the regular exercise per time?", and "What kind of exercise do you regularly have?" (light-, moderate-, vigorous- or very vigorousintensity exercise)". In this study, we added another level, irregular exercise, by defining doing physical activities irregularly, and the average time spent on exercise was less than 10 min per week. In total five levels of exercise were defined (inactive, irregular exercise, low, medium, and high).

Other unhealthy behaviors, including current tobacco smoking and betel-nut chewing, were assessed by three binary indicators $(1=$ yes, $0=$ no $)$.

\section{Health status}

Mental status was measured by the 12-item Chinese Health Questionnaire (CHQ-12) to identify non-psychotic psychiatric disorders in community-dwelling Chinese people $[39,40]$. The items were scored from "not at all" to "much more than usual", and then recorded to 0 (no) and 1 (yes). A total score of CHQ-12, ranging from 0 to 12 , was coded as 1 (i.e., score $\geqq 4$ ) to indicate a high risk for psychiatric disorder (otherwise as 0 ).

Morbidity was evaluated by the number of selfreported chronic diseases investigated at the health screening. The surveyed diseases include type 2 Diabetes mellitus (T2DM), hypertension, hyperlipidemia, kidney disease, cardiac disease, stroke, hepatic disease, gout, osteoporosis, asthma, psychiatric disease, nerve-related disease, intestinal disease, Tuberculosis and metabolic syndrome. A chronic disease was recognized when a participant self-reported to have such disease or take medications for curing the disease $(1=$ yes \& $0=$ no). Some clinical biomarkers were also applied to discover latent patients from those participants self-reporting without three diseases, including 1) hypertension recoded as 1 , if systolic blood pressure $\geqq 140 \mathrm{mmHg}$ or diastolic blood pressure $\geqq 90 \mathrm{mmHg}$; 2) T2DM recoded as 1 , if fasting plasma glucose $\geqq 126 \mathrm{mg} / \mathrm{dL}$; and 3) hyperlipidemia recoded as 1 , if total cholesterol $(\mathrm{CHO}) \geqq 240$ $\mathrm{mg} / \mathrm{dL}$. The range of morbidity could be 0 to 15 .

The use of medications was indexed by counting the number of investigated medications, including refreshing drugs, sleeping pills, sedative medicine, and painkillers ( $1=$ yes $\& 0=$ no for each medication). The score ranged from 0 to 4 .

\section{Social determinants}

Demographic data, including age, gender and education, were incorporated in analytical models as time-constant variables. Age was assessed by the real age (in years) at baseline, and gender was coded as a binary measure. Education was indexed by the years of schooling education. Marital status was time-varying, which was reclassified into two groups: living with a spouse or no spouse.

\section{Time-interval}

This study was a multi-wave longitudinal design, and most predictors were time-varying. We calculated the time interval as the period (in years) between two adjacent participation dates (or the last day of the follow-up year for those enrollees not attending that follow-up screening). In addition, measures of time interval (i.e., Time and Time $^{2}$ ) were also employed to explore the trajectory shape of HRQOL, specifically focusing on both 
physical and mental perspectives (i.e., PCS and MCS scores).

\section{Statistical analyses}

Following a descriptive analysis, the linear mixed-effects modeling (LMM) approach was employed to fit multivariate growth curve models of PCS and MCS using SPSS version 21.0 [41]. The LMM method was chosen because it is suitable for dealing with repeated measures over time [42] and handling subjects with unbalanced design [43]. The percentages of missing data of all covariates for participants at four waves were less than $10 \%$, ranged from 0 to $8.6 \%$ (see Additional file 1). The missing data were not under imputation for less the inconsequential [44]. The time-quadratic interaction terms were added in the models but all were insignificant; thus, those interaction terms were removed from the final models.

At last, only the intercepts and the linear time-slope parameters were retained. PCS and MCS scores were analyzed by three models. Model 0 only included the intercept and linear and quadratic time slopes as predictors to examine the proposed nonlinear growth curve model across time. Model 1 added the focused regular exercise and its interaction with linear time slope to capture total effects of regular exercise on individual changes of HRQOL over time. Finally, Model 2 included all other covariates and their interactions with linear time slope, in order to adjust the associations of regular exercise with HRQOL and to investigate their unique impacts on HRQOL.

\section{Results}

Table 2 exhibits the characteristics of participants at first four waves of LIONS. In contrast to stable PCS scores ranging from 53.0 to 53.6, MCS scores steadily increased from 49.7 to 52.2 during 2006-2014. Of all 6182 enrollees, $54.7 \%$ were women, the average age was 53.5 years. Although the proportion of participants without doing exercise remained around 35.5 to $38.4 \%$, the percentage of participants spending more than 150 min weekly on doing exercise increased obviously from 12.7 to $18.4 \%$.

The characteristics of the participants according to their exercise level at the baseline were examined (see Table 3). There were differences in PCS and MCS across exercise levels, indicating a higher score accompanied with higher exercise level. The "no exercise" and "irregular exercise" groups were more likely to be female, older, lower educated, and having more chronic diseases.

Table 4 shows the trajectories of PCS \& MCS with contemporary regular exercise and other covariates by LMM. For the trajectory of PCS, Model 0 indicated an increasing growth curve $(\beta=0.72, p<0.001)$, but with decelerated growth rate $(\beta=-0.24, p<0.001)$, of PCS score over time. After controlling for regular exercise and other covariates, the deceleration growth curve of PCS score across time was confirmed in Models 1 and 2, because of significantly positive linear time slope $(\beta=$ 0.81, $p<0.001 ; \beta=1.01, p<0.05)$ but negative quadratic time slope $(\beta=-0.25 \&-0.22, p<0.001)$. Consequently, the trajectory of PCS score would increase at the beginning but slow down later on (i.e., a nonlinear, reversed U-shaped curve).

Model 1 exhibited that doing exercise regularly had significantly positive effects on the intercept of PCS score, including those irregular PA. That means those participants who were doing exercise at the beginning had better physical at the beginning. For the effects of regular exercise on the time slope, only doing exercise 150 min weekly would reduce the PCS score over time $(\beta=-0.43, p<0.05)$. With the inclusion of other covariates in Model 2, regular exercise still had significantly positive effects on the initial status of PCS score (at the intercepts), which seemed to be a dose-response relationship based on the levels of effective PA $(\beta=1.20$ $2.55, p<0.001)$. However, regular exercise became insignificant for the changes of PCS score over time $(p>$ 0.05 ), indicating constant effects of regular exercise on PCS score across time.

Model 2 also showed that some covariates had substantial and varying effects on the intercept, but not on the time slope, of PCS score. For time-constant variables, female $(\beta=-0.59, p<0.01)$ or older $\beta=-0.13$, $p<0.001)$ participants had a stably lower initial PCS score over time. Similarly, there were five time-varying covariates with significant effects only on the intercept of PCS score. Both living with a spouse $(\beta=0.62, p<0.05)$ was positively related to the initial status of PCS score. Psychiatric disorder $(\beta=-2.93, p<0.001)$, the number of chronic diseases $(\beta=-0.71, p<0.001)$ and taken medications $(\beta=-2.22, p<0.001)$ had negative effects on the intercept of PCS score. However, these covariates were insignificant across time.

For the trajectory of MCS score, Model 0 showed an increasing growth curve $(\beta=0.79, p<0.001)$ across time. Even if regular exercise and other covariates were controlled in Models 1 and 2, the increasing tendency of MCS score over time still existed, due to significantly positive linear time slope $(\beta=0.95, p<0.001 ; \beta=1.49$, $p<0.01)$. That means the growth curve of MCS score would be an increasing linear trajectory over time.

Model 1 indicated that regular exercise had significantly positive and varying impacts on the intercept of MCS score $(p<0.001)$, except for doing low level exercise $(<150 \mathrm{~min}$ weekly). However, doing exercise regularly had no time effect on MCS score in Model 1.

After controlling for other covariates in Model 2, most levels of regular exercise, except for the low level $(<150$ 
Table 2 Descriptive statistics of HRQOL and covariates at 4 waves

\begin{tabular}{|c|c|c|c|c|}
\hline & $\begin{array}{l}\text { Wave } 1 \\
(N=6182)\end{array}$ & $\begin{array}{l}\text { Wave } 2 \\
(N=4268)\end{array}$ & $\begin{array}{l}\text { Wave } 3 \\
(N=3424)\end{array}$ & $\begin{array}{l}\text { Wave } 4 \\
(N=2407)\end{array}$ \\
\hline PCS score ${ }^{a}$ & $53.0 \pm 7.3$ & $53.6 \pm 6.8$ & $53.4 \pm 7.1$ & $53.2 \pm 7.1$ \\
\hline MCS score ${ }^{a}$ & $49.7 \pm 8.8$ & $50.6 \pm 8.3$ & $50.9 \pm 8.3$ & $52.2 \pm 8.1$ \\
\hline \multicolumn{5}{|l|}{ Time-constant covariates } \\
\hline Gender (female) ${ }^{\mathrm{e}}$ & $54.7 \%$ & & & \\
\hline Age (years) ${ }^{a}$ & $53.5 \pm 13.1$ & & & \\
\hline 30-64 years old & $80.2 \%$ & & & \\
\hline$\geqq 65$ years old & $19.8 \%$ & & & \\
\hline Education (years) ${ }^{a}$ & $10.1 \pm 4.7$ & & & \\
\hline \multicolumn{5}{|l|}{ Time-varying covariates } \\
\hline Time (years) ${ }^{a, b}$ & $0 \pm 0$ & $2.1 \pm 0.3$ & $1.9 \pm 0.9$ & $2.9 \pm 0.5$ \\
\hline \multicolumn{5}{|l|}{ Marital status } \\
\hline Living with the spouse & $89.0 \%$ & $89.4 \%$ & $87.6 \%$ & $87.6 \%$ \\
\hline Living alone & $11.0 \%$ & $10.6 \%$ & $12.4 \%$ & $12.4 \%$ \\
\hline Tobacco smoking (yes) & $18.0 \%$ & $14.0 \%$ & $11.4 \%$ & $11.0 \%$ \\
\hline Betel-nut chewing (yes) & $3.2 \%$ & $2.0 \%$ & $1.4 \%$ & $1.0 \%$ \\
\hline Psychiatric disorder (CHQ-12 $\geqq 4)^{e}$ & $26.3 \%$ & $21.6 \%$ & $18.9 \%$ & $17.2 \%$ \\
\hline \# of chronic diseases ${ }^{\mathrm{a}, \mathrm{c}}$ & $1.6 \pm 1.4$ & $1.7 \pm 1.5$ & $2.0 \pm 1.5$ & $2.0 \pm 1.5$ \\
\hline \# of Medications ${ }^{\mathrm{a}, \mathrm{d}}$ & $0.1 \pm 0.4$ & $0.1 \pm 0.3$ & $0.1 \pm 0.4$ & $0.1 \pm 0.4$ \\
\hline \multicolumn{5}{|l|}{ Regular exercise status } \\
\hline No exercise & $36.9 \%$ & $35.5 \%$ & $38.4 \%$ & $36.7 \%$ \\
\hline Irregular exercise & $40.0 \%$ & $41.7 \%$ & $37.8 \%$ & $35.3 \%$ \\
\hline$<150$ min & $10.4 \%$ & $10.0 \%$ & $9.6 \%$ & $9.5 \%$ \\
\hline 150-299 min & $5.7 \%$ & $5.9 \%$ & $6.8 \%$ & $8.1 \%$ \\
\hline$\geqq 300 \mathrm{~min}$ & $7.0 \%$ & $6.9 \%$ & $7.3 \%$ & $10.3 \%$ \\
\hline
\end{tabular}

Abbreviations: HRQOL Health-related quality of life, PCS Physical component summary, MCS Mental component summary, CHQ-12 12-item Chinese Health Questionnaire, T2DM Type 2 Diabetes mellitus

${ }^{\mathrm{a}}$ Mean \pm SD (standard deviation)

${ }^{\text {b}}$ Time interval between two adjacent waves

'Self-reported and/or diagnosed chronic diseases, including T2DM, hypertension, hyperlipidemia, kidney disease, cardiac disease, stroke, hepatic disease, gout, osteoporosis, asthma, psychiatric disease, nerve-related disease, intestinal disease, Tuberculosis, and metabolic syndrome

$\mathrm{d}_{4}$ pills, including refreshing drugs, sleeping pills, sedative medicine, and painkiller

'The reference group: gender (male), psychiatric disorder (CHQ-12 $=0-3$ )

min per week), were significantly and positively associated with MCS score at the intercept. Additionally, for those significant regular exercise, higher level of effective PA (i.e., $\geqq 300$ min weekly) was related to better initial status of MCS score $(\beta=2.03, p<0.001)$. The effect of irregular physical activities was still significant. However, regular exercise had no significant effect on changing the MCS score over time.

Model 2 exhibited that several covariates had significant and varying impacts on the intercept and linear time slope of MCS score. Age $(\beta=0.09, p<0.001)$ was positively related to the initial status of MCS score, indicating that older participants would perceive better mental QOL constantly. Living with a spouse had a positive effect on the intercept $(\beta=1.77, p<0.001)$ but a negative impact on the time slope $(\beta=-0.40, p<0.05)$ of MCS score. These results revealed that participants living with a spouse had higher MCS score but it would decrease over time. Both psychiatric disorder $(\beta=-6.17, p<0.001)$ and the number of taken medications $(\beta=-2.42, p<0.001)$ only had negative effects on the initial status of MCS score. Namely, participants with the psychiatric disorder or taking more drugs would have lower MCS score at the very beginning. However, morbidities and medications were not significantly on the time slope.

\section{Discussion}

This study used a multiple-wave longitudinal panel data of community-dwelling adults in Northern Taiwan to examine the effects of regular exercise on health-related quality of life over time among community adults. Those with regular exercise showed a significant improving 
Table 3 Similarity of HRQOL scores and covariates by regular exercise status at wave 1

\begin{tabular}{|c|c|c|c|c|c|c|}
\hline \multirow[t]{2}{*}{$N=6182$} & \multicolumn{5}{|c|}{ Regular exercise status } & \multirow[t]{2}{*}{$P$-value } \\
\hline & $\begin{array}{l}\text { No exercise } \\
(n=2141)\end{array}$ & $\begin{array}{l}\text { Irregular exercise } \\
(n=2317)\end{array}$ & $\begin{array}{l}<150 \text { min } \\
(n=601)\end{array}$ & $\begin{array}{l}150-299 \min \\
(n=332)\end{array}$ & $\begin{array}{l}\geqq 300 \text { min } \\
(n=405)\end{array}$ & \\
\hline$\overline{\text { PCS score }} \mathrm{a}^{\mathrm{a}, \mathrm{b}}$ & $52.3 \pm 8.1$ & $52.8 \pm 7.3$ & $54.7 \pm 5.9$ & $54.8 \pm 5.1$ & $54.5 \pm 6.0$ & $<0.001$ \\
\hline PF & $86.8 \pm 21.2$ & $87.2 \pm 19.1$ & $93.1 \pm 12.3$ & $93.4 \pm 11.9$ & $92.8 \pm 13.4$ & $<0.001$ \\
\hline $\mathrm{RP}$ & $79.7 \pm 37.1$ & $82.1 \pm 35.0$ & $86.6 \pm 29.1$ & $87.2 \pm 28.4$ & $88.2 \pm 28.4$ & $<0.001$ \\
\hline BP & $81.8 \pm 21.0$ & $83.9 \pm 18.9$ & $83.7 \pm 18.0$ & $85.7 \pm 16.8$ & $85.3 \pm 18.8$ & $<0.001$ \\
\hline $\mathrm{GH}$ & $62.0 \pm 20.6$ & $65.6 \pm 19.8$ & $68.1 \pm 18.9$ & $69.2 \pm 17.6$ & $70.3 \pm 18.7$ & $<0.001$ \\
\hline MCS score $e^{a, b}$ & $48.5 \pm 9.3$ & $50.2 \pm 8.7$ & $49.1 \pm 8.2$ & $50.9 \pm 7.2$ & $51.9 \pm 8.2$ & $<0.001$ \\
\hline VT & $63.1 \pm 18.8$ & $67.7 \pm 18.1$ & $67.2 \pm 16.2$ & $70.7 \pm 17.0$ & $73.7 \pm 17.7$ & $<0.001$ \\
\hline SF & $84.8 \pm 18.4$ & $86.7 \pm 15.8$ & $87.2 \pm 14.1$ & $89.2 \pm 13.0$ & $89.5 \pm 14.5$ & $<0.001$ \\
\hline RE & $82.9 \pm 34.9$ & $85.6 \pm 32.5$ & $85.1 \pm 31.3$ & $89.1 \pm 28.5$ & $88.6 \pm 28.0$ & 0.001 \\
\hline $\mathrm{MH}$ & $69.9 \pm 17.5$ & $73.1 \pm 16.6$ & $72.1 \pm 16.0$ & $74.4 \pm 15.0$ & $77.3 \pm 16.9$ & $<0.001$ \\
\hline Gender (female) ${ }^{c}$ & $56.1 \%$ & $58.9 \%$ & $42.9 \%$ & $48.8 \%$ & $45.9 \%$ & $<0.001$ \\
\hline Age (years) ${ }^{a, b, c}$ & $51.1 \pm 12.4$ & $57.3 \pm 13.6$ & $46.2 \pm 9.4$ & $51.1 \pm 10.0$ & $55.8 \pm 11.0$ & $<0.001$ \\
\hline 30-64 years old & $85.3 \%$ & $70.9 \%$ & $96.7 \%$ & $90.7 \%$ & $80.2 \%$ & $<0.001$ \\
\hline$\geqq 65$ years old & $14.7 \%$ & $29.1 \%$ & $3.3 \%$ & $9.3 \%$ & $19.8 \%$ & \\
\hline Education (years) ${ }^{a, b}$ & $9.7 \pm 4.6$ & $9.4 \pm 4.8$ & $12.9 \pm 3.3$ & $11.8 \pm 4.0$ & $10.5 \pm 4.1$ & $<0.001$ \\
\hline \multicolumn{7}{|l|}{ Marital status ${ }^{d}$} \\
\hline Living with the spouse & $87.4 \%$ & $90.0 \%$ & $87.6 \%$ & $91.8 \%$ & $90.1 \%$ & 0.016 \\
\hline Living alone & $12.6 \%$ & $10.0 \%$ & $12.4 \%$ & $8.2 \%$ & $9.9 \%$ & \\
\hline Tobacco smoking (yes) ${ }^{c}$ & $24.0 \%$ & $12.3 \%$ & $21.1 \%$ & $15.4 \%$ & $15.6 \%$ & $<0.001$ \\
\hline Alcohol drinking (yes) ${ }^{c}$ & $13.0 \%$ & $9.5 \%$ & $19.5 \%$ & $14.2 \%$ & $13.5 \%$ & $<0.001$ \\
\hline Betel-nut chewing (yes) ${ }^{c}$ & $5.1 \%$ & $1.2 \%$ & $5.3 \%$ & $2.4 \%$ & $2.2 \%$ & $<0.001$ \\
\hline Psychiatric disorder (CHQ-12 $\geqq 4)^{c}$ & $29.8 \%$ & $25.3 \%$ & $24.4 \%$ & $22.5 \%$ & $19.7 \%$ & $<0.001$ \\
\hline \# of chronic diseases ${ }^{\mathrm{a}, \mathrm{b}, \mathrm{d}}$ & $1.64 \pm 1.44$ & $1.77 \pm 1.51$ & $1.32 \pm 1.26$ & $1.39 \pm 1.28$ & $1.58 \pm 1.30$ & $<0.001$ \\
\hline \# of Medications $s^{\mathrm{a}, \mathrm{b}, \mathrm{e}}$ & $0.11 \pm 0.39$ & $0.10 \pm 0.36$ & $0.08 \pm 0.33$ & $0.07 \pm 0.29$ & $0.10 \pm 0.35$ & 0.425 \\
\hline
\end{tabular}

Abbreviations: $H R Q O L$ Health-related quality of life, $P C S$ Physical component summary, PF Physical functioning, RP Role limitations due to physical problems, $B P$ Bodily pain, GH General health, MCS Mental component summary, VT Vitality, SF Social functioning, RE Role emotional, MH Mental health, CHQ-12 12-item Chinese Health Questionnaire, T2DM Type 2 Diabetes mellitus

${ }^{\text {a }}$ Mean \pm SD (standard deviation); ${ }^{\text {b }}$ by ANOVA; by ${ }^{c}$ Chi-square test

d Self-reported and/or diagnosed chronic diseases, including T2DM, hypertension, hyperlipidemia, kidney disease, cardiac disease, stroke, hepatic disease, gout, osteoporosis, asthma, psychiatric disease, nerve-related disease, intestinal disease, Tuberculosis, and metabolic syndrome

e 4 pills, including refreshing drugs, sleeping pills, sedative medicine, and painkiller

effect on physical health than those without doing exercise at the intercepts, especially if the regular exercise time was more than $150 \mathrm{~min}$ a week. Regular exercise also showed positive effects (at the intercepts) on mental health if the exercise time was $150 \mathrm{~min}$ or more in a week. However, the regular exercise effects were not significant over time (at time slope) on either physical or mental health scores. In addition, physical health and mental health improved over time, but the time effect would offset a little bit on physical health. Moreover, being female, increasing age, living alone, or poorer health status were related to lower physical health, but drinking alcohol was related to better physical health. The participants who were younger, lived alone or had poorer health status would have worse mental health.
The present research demonstrates that compared to non-exercising almost all levels of PA had positive effects on PCS and MCS. These results were consistent with the literature that usually speculated the varying positive associations of exercise/PA with overall quality-of-life $[16,18,45,46]$, PCS $[10,30$, 46-50], MCS [10, 11, 30, 47], or subjective well-being $[27,28,51,52]$.

In our study, $76.9 \%$ of our participants did not have exercise habits or did not meet the recommendation criteria. Previous research indicated that $86 \%$ of Taiwanese did not meet the national recommendation [28]. However, the present research showed that even irregular levels of PA lower than currently recommended for beneficial health effects [38] were also related to the 
Table 4 Trajectories of HRQOL by regular exercise and covariates ( $n=6182$ persons, 16,281 observations)

\begin{tabular}{|c|c|c|c|c|c|c|}
\hline \multirow[t]{2}{*}{ Covariates } & \multicolumn{3}{|l|}{ PCS score } & \multicolumn{3}{|l|}{ MCS score } \\
\hline & Model 0 & Model 1 & Model 2 & Model 0 & Model 1 & Model 2 \\
\hline \multicolumn{7}{|l|}{ Fixed effects } \\
\hline \multicolumn{7}{|l|}{ For intercept } \\
\hline Intercept & $\begin{array}{l}53.02^{* * *} \\
(52.83 \sim 53.20)\end{array}$ & $\begin{array}{l}52.25^{* * *} \\
(51.96 \sim 52.54)\end{array}$ & $\begin{array}{l}60.67^{* * *} \\
(59.26 \sim 62.07)\end{array}$ & $\begin{array}{l}49.74^{* * *} \\
(49.51 \sim 49.96)\end{array}$ & $\begin{array}{l}48.52^{* * *} \\
(48.17 \sim 48.87)\end{array}$ & $44.51^{* * *}(42.80 \sim 46.21)$ \\
\hline Gender (female) $^{d}$ & & & $\begin{array}{l}-0.59^{* *} \\
(-0.99 \sim-0.19)\end{array}$ & & & $-0.26(-0.74 \sim 0.22)$ \\
\hline Education (years) & & & $0.03(-0.01 \sim 0.08)$ & & & $-0.002(-0.06 \sim 0.05)$ \\
\hline AGE (years) & & & $\begin{array}{l}-0.13^{* * *} \\
(-0.15 \sim-0.11)\end{array}$ & & & $0.09^{* * *}(0.07 \sim 0.11)$ \\
\hline \multicolumn{7}{|l|}{ Time-varying covariates } \\
\hline $\begin{array}{l}\text { Living with the } \\
\text { spouse }^{d}\end{array}$ & & & $0.62^{*}(0.09 \sim 1.14)$ & & & $1.77^{* * *}(1.14 \sim 2.40)$ \\
\hline $\begin{array}{l}\text { Tobacco smoking } \\
\text { (yes) }\end{array}$ & & & $0.14(-0.36 \sim 0.64)$ & & & $0.06(-0.54 \sim 0.67)$ \\
\hline $\begin{array}{l}\text { Betel-nut chewing } \\
\text { (yes) }\end{array}$ & & & $-0.18(-1.20 \sim 0.83)$ & & & $-0.32(-1.55 \sim 0.91)$ \\
\hline $\begin{array}{l}\text { Psychiatric } \\
\text { disorder }^{d}\end{array}$ & & & $\begin{array}{l}-2.93^{* * *} \\
(-3.31 \sim-2.54)\end{array}$ & & & $-6.17^{* * *}(-6.64 \sim-5.71)$ \\
\hline $\begin{array}{l}\text { \# of chronic } \\
\text { diseases }^{b}\end{array}$ & & & $\begin{array}{l}-0.71^{* * *} \\
(-0.83 \sim-0.59)\end{array}$ & & & $0.09(-0.05 \sim 0.24)$ \\
\hline \# of Medications ${ }^{\complement}$ & & & $\begin{array}{l}-2.22^{* * *} \\
(-2.68 \sim-1.76)\end{array}$ & & & $-2.42^{* * *}(-2.97 \sim-1.86)$ \\
\hline \multicolumn{7}{|l|}{ Regular exercise status ${ }^{d}$} \\
\hline$\geqq 300 \mathrm{~min}$ & & $\begin{array}{l}2.27^{* * *} \\
(1.57 \sim 2.97)\end{array}$ & $2.54^{* * *}(1.86 \sim 3.22)$ & & $3.39 * * *(2.55 \sim 4.24)$ & $2.03^{* * *}(1.21 \sim 2.85)$ \\
\hline 150-299 min & & $\begin{array}{l}2.68^{* * *} \\
(1.91 \sim 3.45)\end{array}$ & $1.99^{* * *}(1.25 \sim 2.72)$ & & $2.52^{* * *}(1.59 \sim 3.44)$ & $1.55^{* *}(0.65 \sim 2.44)$ \\
\hline$<150$ min & & $\begin{array}{l}2.39^{* * *} \\
(1.78 \sim 3.00)\end{array}$ & $1.24^{* * *}(0.66 \sim 1.83)$ & & $0.71(-0.02 \sim 1.45)$ & $0.65(-0.06 \sim 1.36)$ \\
\hline Irregular exercise & & $\begin{array}{l}0.55^{* *} \\
(0.15 \sim 0.94)\end{array}$ & $1.27^{* * *}(0.89 \sim 1.66)$ & & $1.89^{* * *}(1.41 \sim 2.36)$ & $0.87^{* * *}(0.40 \sim 1.33)$ \\
\hline \multicolumn{7}{|l|}{ For time slope } \\
\hline Time $(\text { years })^{a}$ & $\begin{array}{l}0.72^{* * *} \\
(0.42 \sim 1.03)\end{array}$ & $\begin{array}{l}0.81^{* * *} \\
(0.48 \sim 1.15)\end{array}$ & $1.01 *(0.06 \sim 1.97)$ & $0.79 * * *(0.42 \sim 1.17)$ & $0.95^{* * *}(0.54 \sim 1.35)$ & $1.49^{* *}(0.37 \sim 2.62)$ \\
\hline Time $^{2}$ (years) & $\begin{array}{l}-0.24^{* * *} \\
(-0.35 \sim-0.14)\end{array}$ & $\begin{array}{l}-0.25^{* * *} \\
(-0.36 \sim-0.15)\end{array}$ & $\begin{array}{l}-0.22^{* * *} \\
(-0.33 \sim-0.10)\end{array}$ & $-0.06(-0.19 \sim 0.07)$ & $-0.06(-0.19 \sim 0.07)$ & $-0.05(-0.19 \sim 0.08)$ \\
\hline Gender (female) ${ }^{d}$ & & & $0.02(-0.23 \sim 0.27)$ & & & $-0.14(-0.43 \sim 0.15)$ \\
\hline Education (years) & & & $-0.003(-0.03 \sim 0.03)$ & & & $-0.01(-0.05 \sim 0.02)$ \\
\hline AGE (years) & & & $-0.01(-0.02 \sim 0.003)$ & & & $-0.005(-0.02 \sim 0.01)$ \\
\hline \multicolumn{7}{|l|}{ Time-varying covariates } \\
\hline $\begin{array}{l}\text { Living with the } \\
\text { spouse }^{d}\end{array}$ & & & $-0.09(-0.40 \sim 0.23)$ & & & $-0.40^{*}(-0.77 \sim-0.03)$ \\
\hline $\begin{array}{l}\text { Tobacco smoking } \\
\text { (yes) }\end{array}$ & & & $0.01(-0.32 \sim 0.34)$ & & & $-0.15(-0.54 \sim 0.24)$ \\
\hline $\begin{array}{l}\text { Betel-nut chewing } \\
\text { (yes) }\end{array}$ & & & $0.50(-0.25 \sim 1.25)$ & & & $0.69(-0.21 \sim 1.59)$ \\
\hline $\begin{array}{l}\text { Psychiatric } \\
\text { disorder }^{d}\end{array}$ & & & $0.11(-0.13 \sim 0.35)$ & & & $0.19(-0.09 \sim 0.48)$ \\
\hline \# of 15 diseases $^{\mathrm{b}}$ & & & $0.02(-0.05 \sim 0.10)$ & & & $-0.08(-0.17 \sim 0.005)$ \\
\hline \# of Medications ${ }^{c}$ & & & $0.05(-0.23 \sim 0.32)$ & & & $0.10(-0.22 \sim 0.43)$ \\
\hline
\end{tabular}


Table 4 Trajectories of HRQOL by regular exercise and covariates ( $n=6182$ persons, 16,281 observations) (Continued)

\begin{tabular}{|c|c|c|c|c|c|c|}
\hline \multirow[t]{2}{*}{ Covariates } & \multicolumn{3}{|l|}{ PCS score } & \multicolumn{3}{|l|}{ MCS score } \\
\hline & Model 0 & Model 1 & Model 2 & Model 0 & Model 1 & Model 2 \\
\hline \multicolumn{7}{|c|}{ Regular exercise status $^{\mathrm{d}}$} \\
\hline$\geqq 300 \mathrm{~min}$ & & $\begin{array}{l}-0.02 \\
(-0.41 \sim 0.37)\end{array}$ & $0.03(-0.36 \sim 0.41)$ & & $-0.43(-0.89 \sim 0.04)$ & $0.001(-0.46 \sim 0.47)$ \\
\hline 150-299 min & & $\begin{array}{l}-0.24 \\
(-0.67 \sim 0.18)\end{array}$ & $0.08(-0.34 \sim 0.50)$ & & $\begin{array}{l}-0.50 \\
(-1.01 \sim-0.001)\end{array}$ & $-0.11(-0.62 \sim 0.39)$ \\
\hline$<150$ min & & $\begin{array}{l}-0.43^{*} \\
(-0.78 \sim-0.08)\end{array}$ & $0.03(-0.32 \sim 0.38)$ & & $-0.10(-0.53 \sim 0.32)$ & $-0.12(-0.54 \sim 0.30)$ \\
\hline Irregular exercise & & $\begin{array}{l}-0.03 \\
(-0.26 \sim 0.20)\end{array}$ & $0.04(-0.19 \sim 0.28)$ & & $-0.21(-0.48 \sim 0.07)$ & $0.07(-0.20 \sim 0.35)$ \\
\hline \multicolumn{7}{|l|}{ Random effects } \\
\hline Variance (Wave 1) & $53.76^{* * *}$ & $53.01^{* * *}$ & $44.84^{* * *}$ & $78.02^{* * *}$ & $77.07^{* * *}$ & $65.81^{* * *}$ \\
\hline Variance (Wave 2) & $29.31^{* * *}$ & $29.39^{* * *}$ & $27.81^{* * *}$ & $50.18^{* * *}$ & $50.46^{* * *}$ & $48.30^{* * *}$ \\
\hline Variance (Wave 3) & $35.20^{* * *}$ & $35.03^{* * *}$ & $32.82^{* * *}$ & $51.28^{* * *}$ & $50.76^{* * *}$ & $47.63^{* * *}$ \\
\hline Variance (Wave 4) & $23.73^{* * *}$ & $23.85^{* * *}$ & $26.37^{* * *}$ & $34.90^{* * *}$ & $35.25^{* * *}$ & $37.01^{* * *}$ \\
\hline Variance (Time) & $4.25^{* * *}$ & $4.06^{* * *}$ & $2.59^{* * *}$ & $4.60^{* * *}$ & $4.44^{* * *}$ & $2.80^{* * *}$ \\
\hline$-2 L L$ & $103,650.8$ & $102,351.7$ & $94,864.7$ & $109,273.9$ & $107,958.8$ & $100,337.1$ \\
\hline AIC & $103,660.8$ & $102,361.7$ & $94,874.7$ & $109,283.9$ & $107,968.8$ & $100,347.1$ \\
\hline $\mathrm{BIC}$ & $103,699.0$ & $102,399.9$ & $94,912.6$ & $109,322.1$ & $108,007.0$ & $100,385.0$ \\
\hline
\end{tabular}

Abbreviations: HRQOL Health-related quality of life, PCS Physical component summary, MCS Mental component summary, T2DM type 2 Diabetes mellitus, $-2 L L-2$ restricted log-likelihood, AIC Akaike's information criterion, BIC Schwarz's Bayesian criterion

${ }^{*} p<0.05 ;{ }^{* *} p<0.01 ;{ }^{* * *} p<0.001$

${ }^{a}$ Time interval between two adjacent waves

${ }^{b}$ Self-reported and/or diagnosed chronic diseases, including T2DM, hypertension, hyperlipidemia, kidney disease, cardiac disease, stroke, hepatic disease, gout, osteoporosis, asthma, psychiatric disease, nerve-related disease, intestinal disease, Tuberculosis, and metabolic syndrome

'4 pills, including refreshing drugs, sleeping pills, sedative medicine, and painkiller

${ }^{\mathrm{d}}$ The reference group: gender (male), marital status (living alone), psychiatric disorder (CHQ-12<4), regular exercise status (no exercise)

improvement in PCS. Previous research suggested the positive association of light-intensity PA to different aspects of well-being [28], and the low PA level (40-179 min per week) [47] or even irregular excise [46] still had significantly positive effects on PCS. Therefore, doing exercise regularly would be beneficial for improving physical health, no matter the amount and intensity of PA. Further, this study found the insignificant impact of PA on the linear time slope, indicating that the effects of PA on HRQOL would be constant over time. A curvilinear trend between PA levels and HRQOL, particularly physical functioning and vitality in previous study [47], was not discovered in this study. It is possibly attributed to using different measures of PA, or missing some confounders to physical health.

Regarding to the PA effect on mental health, although the level of $<150$ min was insignificant, further analyses of MCS subscales (see Additional file 2) revealed that it had significantly positive effects on three domains, including vitality, social functioning and mental health. Wicker and Frick [27] suggested that light-intensity PA would be beneficial for improving individuals' mental health too. However, some past research found the insignificant relationship between PA and role limitations due to emotional problems $[11,50]$. Therefore, all levels of PA would have varying influences on MCS and its domains, including irregular exercise. Besides, our results support the conclusion that changes in HRQOL were dose dependent on the amount of PA [10, 18, 46-49].

The trajectory of physical health would increase at first and decrease after a period of time, consistent with the literature. Previous studies have found the trajectories of HRQOL for chronic diseases may be improved by medical interventions [53-55]. Moreover, this study found the aging effect would offset the physical health in quality-of-life, consistent with previous research [56]. The trajectory of mental health was also found to be positively related to time linear effect as previous research $[57,58]$. The time effect to improve mental health can be explained by the socioemotional selectivity theory [59]. Increasing age will limit the future prospections of people and then make them focusing on positive information and seeking for the emotional satisfaction $[60,61]$, and thus people would employ various coping strategies to focus on potential positive outcomes of unfavorable events such as chronic diseases or disabilities.

Previous studies usually contended that women had worse HRQOL than men in general or in specific domains, 
even after controlling for sociodemographic characteristics, economic status and chronic diseases [20, 62-64]. However, our results indicated that female adults only had significantly lower PCS score. The insignificant gender difference in MCS score could be attributed to the inclusion of psychiatric/mental disorder and PA/exercise in the models, which would reduce the direct effect of gender on HRQOL, especially MCS. Previous studies concluded that psychiatric disorder (e.g., anxiety, depression) was more prevalent in women and most important predictor of MCS score [25], and PA had larger positive effects on MCS for women $[30,65]$. This study discovered that chronic diseases had negative impacts on PCS score, consistent with previous literature. However, the relationship between chronic diseases and MCS score was found to be nonlinear, which could be partially explained by psychological adjustment to chronic diseases [59,60]. Namely, the positivity effect of psychological adjustment could be offset by the cumulative burden of chronic diseases over time, resulting in the curvilinear association of MCS score with chronic diseases. Additionally, only one unhealthy behavior (i.e., alcohol drinking) was positively related to PCS score, which was supported by some studies [24, 66] but contrary to other research [23]; nevertheless, other unhealthy behaviors had insignificant and inconsistent impacts on HRQOL. These results could be attributed to binary non-quantity measures [67] and small cases with risky behaviors (e.g., betel-nut chewing).

\section{Study limitations}

This study could be improved in several ways. First, although this study was based on a sizable sample with four repeated observations during 2006-2014, the sample could not be generalized to the district or other populations. Further replications with data collected in Taiwan and other Asian countries would be required to extend the generalizability of our findings. Second, self-reported exercise data could be refined by the adoption of objective instruments, which may reduce measurement errors (i.e., potential overlapping between self-reported data and subjective HRQOL) and avoid incorrect statistical inference $[18,28,52]$. Third, the intensity of regular exercise and all the forms of physical activity can be measured by metabolic equivalents (METS) for a better measure of physical activity. Fourth, a refined model with fewer significant covariates but more interaction terms of PA and time could generate more accurate effects of PA on the trajectory of HRQOL over time. Fourth, alcohol drinking behavior was not included in the model, because the amount of alcohol drinking was not available. Alcohol drinking may be a confounder to HRQOL. In fact, we tried to put alcohol drinking (yes/no) in the models, and it showed a positive effect on PCS at the intercept but non-significant on the time slope or on MCS. A more detailed alcohol intake is suggested to examine the longitudinal effect of alcohol drinking on HRQOL. Fifth, there were attritions in the follow-ups. Only $1.5 \% \sim 4.2 \%$ of the non-participants because of death or loss in the follow-ups. Most of the nonparticipants (more than 95\%) did receive the invitation in the next following waves and considered or agreed to participate in the survey, but eventually they did not show up. We further compared the baseline characteristics between the participants and non-participants, and the participants were more likely to be healthier than the non-participants (please see Additional file 3). Therefore, the sample may be over-estimated the health and healthy lifestyle from the population.

\section{Conclusions}

This study found the positive effects of doing regular exercise on HRQOL to the general population in Taiwan, and the dose-response relationship of regular exercise on HRQOL was found. Regular exercise improves physical and mental dimensions of HRQOL if the cumulative time of exercise is $150 \mathrm{~min}$ or more per week. Even though an irregular exercise (less than 150 min a week) would be beneficial for perceived physical health. We suggest that a regular exercise habit should be built up and promoted by community-based healthcare organizations and the government, and cumulating the exercise time for at least $150 \mathrm{~min}$ per week is even better. We also suggest that future research should identify the long-term effects of various types, intensities and time amount of PA via objective measurement on various domains of HRQOL, as they could be critical to the design of effective and specific health promotion plans for improving HRQOL of community-dwelling people.

\section{Supplementary information}

Supplementary information accompanies this paper at https://doi.org/10. 1186/s12889-019-7662-8.

Additional file 1. Missing data information of $\mathrm{HRQOL}$ and covariates at 4 waves.

Additional file 2. Trajectories of HRQOL (i.e., mental scores) by regular exercise and covariates ( $n=6182$ persons, 16,281 observations).

Additional file 3. Comparisons between non-participation and participation at Wave 2 - Wave4 by predictors at baseline.

\section{Abbreviations}

CHO: Total cholesterol; CHQ-12: 12-item Chinese Health Questionnaire; HRQOL: Health-related quality of life; LIONS: Landseed Integrated Outreaching Neighborhood Screening; MCS: Mental component summary; METS: Metabolic equivalents; PA: Physical activity; PCS: Physical component summary; QOL: Quality of life; SF-36: Medical Outcomes Study short-form 36item; T2DM: Type 2 Diabetes mellitus

\section{Acknowledgments}

We gratefully acknowledge the Landseed International Hospital, colleagues of Division of Health Management and all volunteers for their great helps in the collection and provision of longitudinal data and in conducting this study. 


\section{Authors' contributions}

HCC conceived and designed the LIONS study, interpreted data, and drafted the manuscript. JL designed the LIONS study, supervised the manuscript and approved the final version. $\mathrm{HCH}$ contributed to the conceptualization of the study and design, supervised data analyses and interpretation, revised the manuscript, and approved the final version. SKL contributed to the conceptualization and design of the study, interpreted data, and commented the manuscript. THC undertook the literature review and data analyses, and contributed to the preparation and revision of the manuscript. SHL performed to collect data and assist the interpretation of findings. All authors read and approved the final manuscript.

\section{Funding}

This research did not receive any specific grant from funding agencies in the public, commercial, or not-for-profit sectors.

\section{Availability of data and materials}

The data that support the findings of this study are available from the Landseed International Hospital but restrictions apply to the availability of these data, which were used under license/permission for the current study, and so are not publicly available. However, data are available with permission of the Landseed International Hospital, but can only be accessed in the Landseed International Hospital (currently without the virtual access option).

\section{Ethics approval and consent to participate}

Complying with the 1964 Helsinki declaration \& later amendments and local legislations, this study was approved by the Institutional Review Board of Landseed International Hospital (IRB-16-010-C0). All the participants signed an informed consent to release their data for this study.

\section{Consent for publication}

The consent forms are archived in Landseed International Hospital.

\section{Competing interests}

The authors declare that they have no competing interests.

\section{Author details}

'Division of Family Medicine, Department of Community Medicine, Landseed International Hospital, No.77, Guangtai Rd., Pingzhen Dist., Taoyuan 32449, Taiwan, Republic of China. ${ }^{2}$ Department of Health Care Management, Chang Gung University, No.259, Wen-Hwa 1st Rd., Kwei-Shan Dist., Taoyuan 33302, Taiwan, Republic of China. ${ }^{3}$ Department of Health Management and Policy, School of Public Health, University of Michigan, 1415 Washington Heights, M3007, SPH II, Ann Arbor, MI 48109, USA. ${ }^{4}$ School of Public Health, Taipei Medical University, No.250, Wuxing Street, Taipei 11031, Taiwan, Republic of China. ${ }^{5}$ Research Center of Health Equity, College of Public Health, Taipei Medical University, No.250, Wuxing Street, Taipei 11031, Taiwan, Republic of China. 'Landseed Sports Medicine Center, Landseed International Hospital, No.77, Guangtai Rd., Pingzhen Dist., Taoyuan 32449, Taiwan, Republic of China. ${ }^{7}$ Department of Medical Education, Research and Quality Management, Landseed International Hospital, No.77, Guangtai Rd., Pingzhen Dist., Taoyuan 32449, Taiwan, Republic of China. ${ }^{8}$ Division of Health Care Management, Department of Community Medicine, Landseed International Hospital, No.77, Guangtai Rd., Pingzhen Dist., Taoyuan 32449, Taiwan, Republic of China.

Received: 20 May 2019 Accepted: 20 September 2019 Published online: 23 October 2019

\section{References}

1. National Institutes of Health (NIH) Consensus Conference. Physical activity and cardiovascular health: $\mathrm{NIH}$ consensus development panel on physical activity and cardiovascular health. JAMA. 1996;276(3):241-6. https://doi.org/ 10.1001/jama.1996.03540030075036.

2. World Health Organization (WHO). Global recommendations on physical activity for health. Geneva: WHO; 2010. Retrieved from https://apps.who.int/ iris/bitstream/handle/10665/44399/9789241599979_eng.pdf?sequence=1. Accessed 14 Jan 2019

3. Goncalves A, Florencio G, Silva M, Cobucci R, Giraldo P, Cote N. Effects of physical activity on breast cancer prevention: a systematic review. J Phys Act Health. 2014;11(2):445-54. https://doi.org/10.1123/jpah.2011-0316.
4. Motl RW, McAuley E. Physical activity, disability, and quality of life in older adults. Phys Med Rehabil Clin N Am. 2010;21(2):299-308. https://doi.org/10. 1016/j.pmr.2009.12.006.

5. Steinmo S, Hagger-Johnson G, Shahab L. Bidirectional association between mental health and physical activity in older adults: Whitehall II prospective cohort study. Prev Med. 2014;66:74-9. https://doi.org/10.1016/j.ypmed.2014. 06.005.

6. Lee Y, Park K. Does physical activity moderate the association between depressive symptoms and disability in older adults? Int I Geriatr Psychiatry. 2008;23(3):249-56. https://doi.org/10.1002/gps.1870.

7. Baker LD, Frank LL, Foster-Schubert K, et al. Effects of aerobic exercise on mild cognitive impairment: a controlled trial. Arch Neurol. 2010;67(1):71-9. https://doi.org/10.1001/archneurol.2009.307.

8. Bize R, Johnson JA, Plotnikoff RC. Physical activity level and health-related quality of life in the general adult population: a systematic review. Prev Med. 2007:45(6):401-15. https://doi.org/10.1016/j.ypmed.2007.07.017.

9. Chai W, Nigg CR, Pagano IS, Motl RW, Horwath C, Dishman RK. Associations of quality of life with physical activity, fruit and vegetable consumption, and physical inactivity in a free living, multiethnic population in Hawaii: a longitudinal study. Int J Behav Nutr Phys Act. 2010;7:83-8. https://doi.org/10. 1186/1479-5868-7-83.

10. Martin CK, Church TS, Thompson AM, Earnest CP, Blair SN. Exercise dose and quality of life: a randomized controlled trial. Arch Intern Med. 2009; 169(3):269-78. https://doi.org/10.1001/archinternmed.2008.545.

11. Wendel-Vos GCW, Schuit AJ, Tijhuis MA, Kromhout D. Leisure time physical activity and health-related quality of life: cross-sectional and longitudina associations. Qual Life Res. 2004;13(3):667-77. https://doi.org/10.1023/B: QURE.0000021313.51397.33.

12. Office of Disease Prevention and Health Promotion (ODPHP), Department of Health and Human Services (DHHS). Healthy people 2020 framework. Rockville: U.S. DHHS; 2010. Retrieved from https://www.healthypeople.gov/ sites/default/files/HP2020Framework.pdf. Accessed 14 Jan 2019

13. Hennessy CH, Moriarty DG, Zack MM, Scherr PA, Brackbill R. Measuring health-related quality of life for public health surveillance. Public Health Rep. 1994;109(5):665-72.

14. Cooper JK, Kohlmann T, Michael JA, et al. Health outcomes: new quality measure for Medicare. Int J Qual Health Care. 2001;13(1):9-16. https://doi. org/10.1093/intqhe/13.1.9.

15. Eriksson MK, Hagberg L, Lindholm L, et al. Quality of life and cost-effectiveness of a 3-year trial of lifestyle intervention in primary health care. Arch Intern Med. 2010;170(16):1470-9. https://doi.org/10.1001/archinternmed.2010.301.

16. Rakhshani T, Shojaiezadeh D, Lankarani KB, Rakhshani F, Kaveh MH, Zare N. The association of health-promoting lifestyle with quality of life among the Iranian elderly. Iran Red Crescent Med J. 2014;16(9):1-6. https://doi.org/10. 5812/ircmj.18404.

17. Oh SH, Kim DK, Lee SU, Jung SH, Lee SY. Association between exercise type and quality of life in a community-dwelling older people: a cross-sectional study. PLoS One. 2017;12(12):1-11. https://doi.org/10.1371/journal.pone. 0188335.

18. Anokye NK, Trueman P, Green C, Pavey TG, Taylor RS. Physical activity and health related quality of life. BMC Public Health. 2012;12:624-31. https://doi. org/10.1186/1471-2458-12-624.

19. Lubetkin El, Jia H, Franks P, Gold MR. Relationship among sociodemographic factors, clinical conditions, and health-related quality of life: examining the EQ-5D in the U.S. general population. Qual Life Res. 2005;14(10):2187-96. https://doi.org/10.1007/s11136-005-8028-5.

20. Hsu HC. Gender differences in health-related quality of life among the elderly in Taiwan. Asian J Health Inform Sci. 2007;1(4):366-76 Retrieved from https://asiair.asia.edu.tw/bitstream/310904400/2059/1/01-his06020.pdf. Accessed 15 Dec 2018.

21. Chen $Y$, Hicks A, While AE. Quality of life and related factors: a questionnaire survey of older people living alone in Mainland China. Qual Life Res. 2014; 23(5):1593-602. https://doi.org/10.1007/s11136-013-0587-2.

22. Coste J, Quinquis L, D'Almeida S, Audureau E. Smoking and health-related quality of life in the general population: independent relationships and large differences according to patterns and quantity of smoking and to gender. PLoS One. 2014;9(3):e91562. https://doi.org/10.1371/journal.pone. 0091562.

23. Chen CY, Storr CL. Alcohol use and health-related quality of life among youth in Taiwan. J Adolesc Health. 2006;39(5):752. https://doi.org/10.1016/j. jadohealth.2006.04.019. 
24. Wang TF, Chou C, Yu S. Assessing the effects of oral health-related variables on quality of life in Taiwanese adults. Qual Life Res. 2013;22(4):811-25. https://doi.org/10.1007/s11136-012-0205-8.

25. Saarni SI, Suvisaari J, Sintonen $\mathrm{H}$, et al. Impact of psychiatric disorders on health-related quality of life: general population survey. Br J Psychiatry. 2007; 190:326-32. https://doi.org/10.1192/bjp.bp.106.025106.

26. Kao TW, Lai MS, Tsai TJ, Jan CF, Chie WC, Chen WY. Economic, social, and psychological factors associated with health-related quality of life of chronic hemodialysis patients in northern Taiwan: a multicenter study. Artif Organs. 2009:33(1):61-8. https://doi.org/10.1111/j.1521-1594.2008.00675.x.

27. Wicker P, Frick B. Intensity of physical activity and subjective well-being: an empirical analysis of the WHO recommendations. J Public Health (Oxf). 2017;39(2):e19-26. https://doi.org/10.1093/pubmed/fdw062.

28. Ku PW, Fox KR, Liao Y, Sun WJ, Chen LJ. Prospective associations of objectively assessed physical activity at different intensities with subjective well-being in older adults. Qual Life Res. 2016;25(11):2909-19. https://doi. org/10.1007/s11136-016-1309-3.

29. Buman MP, Hekler EB, Haskell WL, et al. Objective light-intensity physical activity associations with rated health in older adults. Am J Epidemiol. 2010; 172(10):1155-65. https://doi.org/10.1093/aje/kwq249.

30. Tessier S, Vuillemin A, Bertrais S, et al. Association between leisure-time physical activity and health-related quality of life changes over time. Prev Med. 2007:44(3):202-8. https://doi.org/10.1016/j.ypmed.2006.11.012.

31. Holistila A, Mänty M, Rahkonen O, Lahelma E, Lahti J. Changes in leisuretime physical activity health functioning: a follow-up study. Scan J Med Sci Sports. 2017;27:1785-92.

32. Chang HC, Kung YY, Hsieh CF, Hsiung LHY, Chang SH, Chen THH. Biological risk factors relevant to chronic disease in three ethnic groups in Taiwan: results from Li-Shin outreaching neighborhood screening (Lions A1). Ethnic Dis. 2008;18(2):228-34.

33. Lu JFR, Tseng HM, Tsai YJ. Assessment of health-related quality of life in Taiwan (I): development and psychometric testing of SF-36 Taiwan version. Taiwan J Public Health. 2003;22(6):501-11. [in Chinese]. https://doi.org/10. 6288/TJPH2003-22-06-09.

34. Ware JE, Snow KK, Kosinski M, et al. SF-36 health survey: manual and interpretation guide. Boston: The Health Institute, New England Medical Center, Nimrod Press; 1993. Retrieved from https://www.researchgate.net/ publication/313050850_SF-36_Health_Survey_Manual_Interpretation_Guide. Accessed 1 June 2018

35. Tseng HM, Lu JFR, Tsai YJ. Assessment of health-related quality of life in Taiwan (II): norming and validation of SF-36 Taiwan version. Taiwan J Public Health. 2003;22(6):512-8 [in Chinese] Retrieved from https://www. researchgate.net/publication/279621013_Assessment_of_health-related_ quality_of_life_in_Taiwan_II_Norming_and_validation_of_SF-36_Taiwan_ version. Accessed 1 June 2018.

36. Ware JE, Sherbourne CD. The MOS 36-item short-form health survey (SF-36): I. conceptual framework and item selection. Med Care. 1992;30(6):473-83. https://doi.org/10.1097/00005650-199206000-00002.

37. Ware JE, Kosinski M, Keller SK. SF-36 physical and mental health summary scales: a user's manual. Boston: The Health Institute, New England Medical Center, Nimrod Press; 1994. Retrieved from https://www.researchgate.net/ profile/John_Ware/publication/292390260_SF-36_Physical_and_Mental_ Health_Summary_Scales_a_User's_Manual/links/5af580264585157136 caee31/SF-36-Physical-and-Mental-Health-Summary-Scales-a-Users-Manual. pdf. Accessed 1 June 2018

38. Office of Disease Prevention and Health Promotion (ODPHP), Department of Health and Human Services (DHHS). 2008 physical activity guidelines for Americans. Rockville: U.S. DHHS; 2008. Retrieved from https://health.gov/ paguidelines/pdf/paguide.pdf. Accessed 1 June 2018

39. Cheng TA, Williams $P$. The design and development of a screening questionnaire (CHQ) for use in community studies of mental disorders in Taiwan. Psychol Med. 1986;16(2):415-22. https://doi.org/10.1017/S0033291700009247.

40. Chong MY, Wilkinson G. Validation of 30- and 12-item versions of the Chinese health questionnaire $(\mathrm{CHQ})$ in patients admitted for general health screening. Psychol Med. 1989;19(2):495-505. https://doi.org/10.1017/S0033291700012526.

41. IBM Corporation. IBM SPSS statistics 21.0. Armonk: IBM Corporation; 2012.

42. Laird NM, Ware $\mathrm{JH}$. Random-effects models for longitudinal data. Biometrics. 1982;38(4):963-74. https://doi.org/10.2307/2529876.

43. Vonesh EF, Carter RL. Mixed-effects nonlinear regression for unbalanced repeated measures. Biometrics. 1992;48(1):1-17. https://doi.org/10.2307/ 2532734.
44. Bennett DA. How can I deal with missing data in my study? Aust N Z J Public Health. 2001;25(5):464-9. https://doi.org/10.1111/j.1467-842X.2001. tb00294.x.

45. Mura G, Sancassiani F, Migliaccio GM, Collu G, Carta MG. The association between different kinds of exercise and quality of life in the long term: results of a randomized controlled trial on the elderly. Clin Pract Epidemiol Ment Health. 2014;10:36-41. https://doi.org/10.2174/1745017901410010036.

46. Vuillemin A, Boini S, Bertrais S, Tessier S, Oppert JM, Hercberg S, Guillemin F, Briancon S. Leisure time physical activity and health-related quality of life. Prev Med. 2005;41:562-9.

47. Heesch KC, van Uffelen JGZ, van Gellecum YR, Brown WJ. Dose-response relationships between physical activity, walking and health-related quality of life in mid-age and older women. J Epidemiol Community Health. 2012; 66(8):670-7. https://doi.org/10.1136/jech-2011-200850.

48. Eime R, Harvey J, Payne W. Dose-response of women's health-related quality of life (HRQoL) and life satisfaction to physical activity. J Phys Act Health. 2014;11(2):330-8. https://doi.org/10.1123/jpah.2012-0073.

49. Evangelista LS, Cacciata M, Stromberg A, Dracup K. Dose-response relationship between exercise intensity, mood states, and quality of life in patients with heart failure. J Cardiovasc Nurs. 2017;32(6):530-7. https://doi. org/10.1097/JCN. 0000000000000407.

50. El-Sobkey SB. Physical activity level and adult Saudi health related quality of life. Open J Ther Rehabil. 2014;2:106-13. https://doi.org/10.4236/ojtr.2014. 23015.

51. Wicker P, Coates D, Breuer C. Physical activity and subjective well-being: the role of time. Eur J Pub Health. 2015;25(5):864-8. https://doi.org/10.1093/ eurpub/ckv053.

52. Hamer M, Stamatakis E. Objectively assessed physical activity, fitness and subjective wellbeing. Ment Health Phys Act. 2010;3(2):67-71. https://doi.org/ 10.1016/j.mhpa.2010.09.001.

53. Sajobi $\Pi$, Wang M, Awosoga $\mathrm{O}$, et al. Trajectories of health-related quality of life in coronary artery disease. Circ Cardiovasc Qual Outcomes. 2018;11(3): e003661. https://doi.org/10.1161/CIRCOUTCOMES.117.003661.

54. Yu H, Gao C, Zhang Y, He R, Zhou L, Liang R. Trajectories of health-related quality of life during the natural history of dementia: a six-wave longitudinal study. Int J Geriatr Psychiatry. 2017;32(9):940-8. https://doi.org/10.1002/gps. 4551

55. Le Grande MR, Elliott PC, Murphy BM, et al. Health-related quality of life trajectories and predictors following coronary artery bypass surgery. Health Qual Life Outcomes. 2006;4:49-61. https://doi.org/10.1186/1477-7525-4-49.

56. Spirduso WW, Cronin DL. Exercise dose-response effects on quality of life and independent living in older adults. Med Sci Sports Exerc. 2001;33(6 Suppl):S598-608. https://doi.org/10.1097/00005768-200106001-00028.

57. Hopman WM, Harrison MB, Coo H, Friedberg E, Buchanan M, VanDenKerkhof EG. Associations between chronic disease, age and physical and mental health status. Chronic Dis Can. 2009;29(3):108-16.

58. Singer MA, Hopman WM, MacKenzie TA. Physical functioning and mental health in patients with chronic medical conditions. Qual Life Res. 1999;8(8): 687-91. https://doi.org/10.1023/A:1008917016998.

59. Carstensen LL. Social and emotional patterns in adulthood: support for socioemotional selectivity theory. Psychol Aging. 1992;7(3):331-8. https:// doi.org/10.1037/0882-7974.7.3.331.

60. Dekker J, de Groot V. Psychological adjustment to chronic disease and rehabilitation: an exploration. Disabil Rehabil. 2018;40(1):116-20. https://doi. org/10.1080/09638288.2016.1247469.

61. de Ridder D, Geenen R, Kuijer R, van Middendorp H. Psychological adjustment to chronic disease. Lancet. 2008;372(9634):246-55. https://doi. org/10.1016/S0140-6736(08)61078-8.

62. Cherepanov D, Palta M, Fryback DG, Robert SA. Gender differences in health-related quality-of-life are partly explained by sociodemographic and socioeconomic variation between adult men and women in the US: evidence from four US nationally representative data sets. Qual Life Res. 2010;19(8):1115-24. https://doi.org/10.1007/s11136-010-9673-x.

63. Hajian-Tilaki K, Heidari B, Hajian-Tilaki A. Are gender differences in healthrelated quality of life attributable to sociodemographic characteristics and chronic disease conditions in elderly people? Int J Prev Med. 2017;8:95. https://doi.org/10.4103/ijpvm.IJPVM_197_16.

64. Singh K, Kondal D, Shivashankar R, et al. Health-related quality of life variations by sociodemographic factors and chronic conditions in three metropolitan cities of South Asia: the CARRS study. BMJ Open. 2017;7(10): e018424. https://doi.org/10.1136/bmjopen-2017-018424. 
65. Morimoto T, Oguma Y, Yamazaki S, Sokejima S, Nakayama T, Fukuhara S. Gender differences in effects of physical activity on quality of life and resource utilization. Qual Life Res. 2006;15(3):537-46. https://doi.org/10.1007/ s11136-005-3033-2.

66. Valencia-Martín JL, Galán I, Guallar-Castillón P, Rodríguez-Artalejo F. Alcohol drinking patterns and health-related quality of life reported in the Spanish adult population. Prev Med. 2013;57(5):703-7. https://doi.org/10.1016/j. ypmed.2013.09.007.

67. Kim K, Kim JS. The association between alcohol consumption patterns and health-related quality of life in a nationally representative sample of south Korean adults. PLoS One. 2015;10(3):e0119245. https://doi.org/10.1371/ journal.pone.0119245.

\section{Publisher's Note}

Springer Nature remains neutral with regard to jurisdictional claims in published maps and institutional affiliations.

- fast, convenient online submission

- thorough peer review by experienced researchers in your field

- rapid publication on acceptance

- support for research data, including large and complex data types

- gold Open Access which fosters wider collaboration and increased citations

- maximum visibility for your research: over $100 \mathrm{M}$ website views per year

At BMC, research is always in progress.

Learn more biomedcentral.com/submissions 